\title{
La foule et l'ordre : gouvernement spatial des conduites dans les meetings électoraux
}

\section{Aïcha Bourad et Fanny Parent}

\section{(2) OpenEdition}

1 Journals

Édition électronique

URL : https://journals.openedition.org/cdg/7041

DOI : $10.4000 /$ cdg.7041

ISSN : 2107-7266

Éditeur

UMR 245 - CESSMA

Référence électronique

Aïcha Bourad et Fanny Parent, « La foule et l'ordre : gouvernement spatial des conduites dans les meetings électoraux », Carnets de géographes [En ligne], 15 | 2021, mis en ligne le 30 avril 2021, consulté le 28 mai 2021. URL : http://journals.openedition.org/cdg/7041 ; DOI : https://doi.org/ 10.4000/cdg.7041

Ce document a été généré automatiquement le 28 mai 2021.

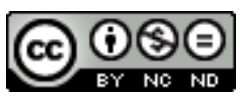

La revue Carnets de géographes est mise à disposition selon les termes de la Licence Creative Commons Attribution - Pas d'Utilisation Commerciale - Pas de Modification 4.0 International. 


\title{
La foule et l'ordre : gouvernement spatial des conduites dans les meetings électoraux
}

\author{
Aïcha Bourad et Fanny Parent
}

\section{Introduction}

Le vendredi 30 mars 2012 à $20 \mathrm{~h} 30$ se déroule à Toulouse le meeting de la campagne présidentielle de la candidate de Lutte Ouvrière (LO), Nathalie Artaud, dans une atmosphère certes familiale liée à l'entre soi militant, mais aussi tendue en raison de la crainte d'une intrusion de militants d'extrême-droite. La salle du meeting se situe très près de l'ancien local des militants du Bloc Identitaire, récemment fermé. $\mathrm{Au}$ moment du meeting les tensions entre ce groupe et les militants d'extrêmegauche toulousains sont fortes et plusieurs militants évoquent le sujet entre eux avant et après le meeting ${ }^{1}$. Pour sécuriser l'événement, les organisateurs ont mis en place un service d'ordre très visible, composé de militants actifs du parti. À l'entrée de la salle cinq militants encadrent les portes et demandent d'ouvrir les sacs; dans la salle, cinq autres membres du service d'ordre sont debout, sur les côtés, de part et d'autre des chaises destinées au public. Ils sont orientés de trois quarts de façon à regarder la salle en ayant la scène dans leur champ de vision. Ils semblent incarner le double rôle de surveillants et de "chauffeurs" de salle. Ils applaudissent ostensiblement, rythmant le discours. Le service d'ordre est organisé en binôme : chaque membre debout est doublé par un acolyte assis, qui occupe la chaise de l'extrémité de chaque rangée, toutes les quatre rangées. Ils échangent leurs postes toutes les 15-20 min. Ce déplacement dans l'espace correspond au fonctionnement et à la vision politique de ce parti, qui milite pour le respect des travailleurs, notamment ceux chargés des tâches pénibles. Il renforce aussi la visibilité et l'omniprésence de ce service d'ordre ${ }^{2}$.

1 Loin des spectaculaires meetings de plein air des candidats des partis les plus puissants dans le champ politique, le meeting que nous venons de décrire n'en est pas moins un dispositif au sens de Michel Foucault c'est-à-dire une stratégie « de rapports de force supportant des types de savoir, et supportés par eux» (1994: 299). Dans cette perspective, cet article ${ }^{3}$ analyse l'organisation pratique des meetings électoraux en tant 
que dispositif inscrivant des rapports de pouvoir dans et par l'espace. Comment l'accueil du public, la façon de l'ordonner et de le gérer physiquement permet de susciter plus ou moins l'adhésion dans le respect de l'ordre public? Au-delà, dans quelle mesure ce dispositif est-t-il aussi le support de l'ordre politique, notamment de la délégation par la représentation politique et participe ainsi au maintien l'ordre social, c'est-à-dire à la distribution et la hiérarchisation des rôles dans la société ?

2 S'ils sont très divers, tant en termes de foules qui s'y déplacent (Mariot, 2001) que de formes qu'ils revêtent, d'ampleur, de localisation en intérieur ou en extérieur, les meetings électoraux constituent l'une des actions de campagne les plus spectaculaires et la plus dispendieuse (François, Phélippeau, 2015). Ils restent un invariant du répertoire d'action politique en France (Abélès, 2010 ; Cossart 2010 ; Mariot, 1995a, 1995b, Neveu, 2011 ; Pourcher, 1990, 2004; Tilly, 1984) comme ailleurs (Baamara, Floderer, Poirier, 2016). Pourtant, à l'exception des travaux d'Hélène Combes sur les meetings de fin de campagne au Mexique (2009), de Claire Sécail sur les meetings des campagnes présidentielles françaises de 2012 et $2017(2016$; 2020) et, toujours pour la campagne présidentielle de 2012, de l'intérêt porté par les chercheurs du groupe SPEL (sociologie politique des Élections) ${ }^{4}$ dont nous faisions partie en 2012 (encadré 1), les travaux de sciences sociales se sont assez peu penchés sur le meeting électoral en tant qu'objet principal de recherche. Cet article propose d'analyser les moyens mobilisés pour mettre en ordre dans l'espace les acteurs présents aux meetings, et les effets de cette organisation spatiale sur les modes de représentation de l'organisation par les militants (Baloge, 2016). Comment l'organisation spatiale, matérielle, du meeting incarne-t-elle la volonté des organisateurs d'attirer du monde (Cossart, 2010) tout en maintenant l'ordre (Sommier, 1993) ? En quoi la gestion physique et symbolique des personnes présentes sur le lieu du meeting participe-t-elle d'une représentation politique au sens à la fois de mise en spectacle mais aussi de matérialisation d'idées politiques? Comment l'organisation spatiale des meetings est-elle utilisée comme mécanisme de contrôle du public et de l'expression politique (Baloge 2016) ? Comment les différences partisanes se traduisent-elles dans ces dispositifs?

3 À la suite de nombreux travaux qui ont défendu l'intérêt de spatialiser l'analyse des mouvements sociaux et des mobilisations collectives (dont Auyero 2005; Ripoll 2005; 2008, Hmed 2008; 2020; Combes, Garibay et Goirand, 2015), l'enjeu de cet article est d'intégrer la dimension spatiale comme paramètre central afin d'appréhender les modes de contrôle du public par les organisateurs du meeting (Baloge, 2016). Penser le meeting comme un dispositif d'encadrement des corps en présence (Foucault, 1975) par un espace, un temps et une ambiance rappelle que l'organisation de l'espace est un moyen de maintien de l'ordre public et social (Merry, 2001 ; Beauguitte, 2019). Aussi, sans nier l'importance des discours et de leurs contenus (Le Bart, 1998 ; Burger, Jacquin, Micheli, 2011) sans nier non plus l'intérêt que présente une sociologie des membres du public des meetings (Combes, 2009), il s'agit ici de montrer que l'organisation spatiale ne constitue pas un élément accessoire ou seulement conjoncturel mais incarne aussi la vision politique de chaque parti. Le meeting est ainsi saisi, dans sa dimension politique, comme un rite institutionnel qui «tend à consacrer ou à légitimer, c'est-à-dire à faire méconnaitre en tant qu'arbitraire et reconnaître en tant que légitime [...] ou, ce qui revient au même, à opérer solennellement, [...], une transgression des limites constitutives de l'ordre social et de l'ordre mental qu'il s'agit de sauvegarder à tous prix » (Bourdieu, Thompson, 2001 : 176). En d'autres termes, il s'agit d'unir un public 
politique et de participer à la naturalisation de la distinction entre professionnels et profanes, représentés et représentants (Gaxie, 2004)5 . Le meeting est aussi saisi comme un dispositif technique de gouvernementalité c'est-à-dire comme faisant partie de «l'ensemble constitué par les institutions, les procédures, analyses et réflexions, les calculs et les tactiques qui permettent d'exercer cette forme bien spécifique, quoique très complexe, de pouvoir, qui a pour cible principale la population, pour forme majeure de savoir l'économie politique, pour instrument technique essentiel les dispositifs de sécurité» (Foucault, 2004: 111-112). La conjugaison de ces deux approches conceptuelles permet ainsi de montrer que le meeting tend à (re)produire la frontière entre représentants et représentés et, par là-même, à (re)produire l'ordre social de la représentation politique. Le meeting est ainsi un rite qui participe avec d'autres (comme le vote) à pacifier la compétition politique tout en l'incarnant, à travers la démonstration subtile d'un rapport de force: donner à voir la capacité (variable) des groupes politiques à mobiliser des forces et à les gouverner ou les discipliner.

En effet, historiquement, les normes qui définissent l'organisation des meetings politiques sont intimement attachées au respect de l'ordre public. Selon Paula Cossart (2010), l'autorisation des réunions publiques est très fortement liée à la capacité d'un groupe politique à assurer le bon déroulement de l'évènement en en contrôlant les participants, notamment par la création des services d'ordre. C'est d'ailleurs la raison pour laquelle un espace clos a été progressivement imposé par les législateurs à mesure que le sens $d u$ bon déroulement de cet évènement politique s'est confondu avec le respect de l'ordre public, afin de contenir la foule et de lui ouvrir des espaces d'expressions normés sans pour autant permettre les débordements et autres désordres. Ce lien entre la répartition du public, son contrôle, et l'espace physique et symbolique de l'expression politique est au cœur de notre propos. L'histoire des meetings en France distingue deux pôles entre lesquels se déploient différentes répartitions du public et différentes modalités de contrôle des conduites des participants. D'un côté, les réunions publiques sont particulièrement privilégiées sous la Troisième République et sous l'Empire, pour des raisons de maintien de l'ordre, parce qu'elles constituent des espaces restreints facilitant le contrôle du public : « la salle de réunion constitue une matérialisation de l'espace public idéal» (Cossart, 2010: 74) ${ }^{6}$. D'un autre côté, dans l'entre-deux-guerres, les réunions publiques laissent place à des affrontements entre partis, parfois très violents. Il s'agit alors moins de convaincre par l'argument et la contradiction que de faire la démonstration de la puissance et de la cohésion du parti, en particulier en contre-manifestant en cas de meeting du parti adverse.

5 Cet article entend ainsi montrer comment les meetings étudiés durant l'élection présidentielle de 2012 se distribuent, en termes d'organisation, sur un continuum qui relie ces deux pôles historiques, même si la pacification générale de l'espace public tend à privilégier le modèle de la réunion publique de la troisième République et la démonstration de force symbolique. Ces questions sont appréhendées sous l'angle de la gestion de l'ordre et du public dans les meetings. Dans un premier temps, il s'agit de mettre en évidence les modalités de construction de l'ordre par la discipline. Les meetings donnent à voir des degrés variés d'encadrements des corps en présence selon des modalités de contraintes et de contrôle imposées de manière plus ou moins directe par les organisateurs. Ces différentes modalités de maintien de l'ordre et leur incarnation dans des services d'ordre donnent à voir, au-delà des nécessités 
organisationnelles, différentes versions de l'espace public idéal selon chaque groupe partisan. Dans un second temps, il s'agit de montrer que ces mises en scène et mises en ordre des corps contribuent à différentes formes de gouvernementalité qui participent à la sacralisation de la politique et matérialisent le coup de force symbolique de la représentation (Bourdieu 1981).

\section{Encadré 1. Contexte et méthodologie de l'enquête}

Cette recherche s'inscrit dans le cadre d'une enquête collective plus large qui a réuni sous la dénomination SPEL (sociologie politique des Élections) des sociologues au niveau national autour de la campagne présidentielle de 2012. Plusieurs sous-groupes spécialisés sur différentes thématiques ont publié les résultats de leurs travaux (SPEL, 2014 ; Courty, Gervais, 2016) ${ }^{7}$. L'enquête réalisée au niveau toulousain, dont nous faisions partie, participait au groupe SPELmeeting. Au sein même de cette petite équipe locale les angles d'analyses choisis étaient variés ${ }^{8}$. L'ensemble des données a été recueilli par le collectif et collectivisé. La présence d'au moins trois observateurs à chaque meeting a permis de multiplier les placements et les angles de vue, offrant ainsi un aperçu plus complet de l'événement en train de se dérouler. Cela a également rendu possible le recueil de nombreuses discussions informelles, qu'elles aient été entendues et notées alors que des sympathisants ou militants discutaient entre eux, ou bien qu'elles aient été provoquées par les enquêteurs avant, pendant ou après le meeting (parfois en se présentant comme enquêteur, parfois comme simple participant). Des négociations ont dans certains cas pu permettre de visiter les lieux en amont du meeting (Front National) ou d'assister à des réunions d'organisation simplement en suivant les militants (Front de Gauche). L'analyse et le traitement des données relatives à la dimension spatiale sont toutefois propres aux autrices de l'article. . Pour chaque meeting analysé dans cet article, au moins l'une d'entre elles a réalisé des observations. Cette étude s'appuie ainsi sur un corpus de huit meetings qui se sont déroulés à Toulouse pendant la campagne présidentielle de 2012, soit la totalité des meetings électoraux ayant eu lieu dans cette ville (figure 1 ).

Nous mobilisons à la fois les enregistrements audio et vidéo et les observations directes des différents temps du meeting (avant, pendant et après), en fonction des contacts et du niveau d'accès que nous sommes parvenus à obtenir dans un temps parfois très court entre l'annonce et l'organisation de l'évènement. En effet, certains meetings étaient prévus et annoncés de longue date, alors que d'autres s'inscrivaient dans la temporalité accélérée des positionnements stratégiques de campagne au fil des jours, des « coups » joués par les adversaires politiques et des éléments fournis par les sondages. Il s'agit donc d'une enquête ethnographique circonscrite temporellement et spatialement au cours de laquelle nous avons porté une attention particulière à la gestion du public et aux services d'ordre. 


\section{Discipliner les corps dans et par l'espace : produire l'ordre (social) dans les meetings}

6 Si le meeting est par définition une réunion ouverte, le degré d'ouverture et les façons de s'ouvrir au public varient selon les groupes politiques (Cossart, 2010). L'organisation spatiale du lieu du meeting (choix et agencement des salles) et la répartition du public en sous-groupes ordonnés par les équipes organisatrices et leurs services d'ordre, militants ou professionnels, proposent des variations dans la discipline des corps - qui est avant tout «l'art des répartitions» des individus dans l'espace selon Michel Foucault (1975: 166) - en échos aux différentes valeurs politiques portées par chaque parti. Cette organisation pratique est aussi une organisation symbolique d'un espace public idéalisé pour et par le groupe partisan organisateur. Cette partie analyse la dimension matérielle comme première façon de mettre en ordre, de trier et de filtrer le public et porte une attention particulière aux différentes pratiques des services d'ordre. Plus ou moins visibles dans l'espace, ces derniers jouent un rôle qui oscille entre deux dynamiques qui renvoient aux deux modalités historiques d'organisation des meetings : service d'accueil pacifié où la question de l'ordre est euphémisée, par opposition au service d'ordre comme organe privilégié de gestion de la menace (indiscipline partisane ou perturbateurs extérieurs).

Tableau 1. Les meetings de la campagne présidentielle de 2012 à Toulouse

\begin{tabular}{|c|c|c|c|}
\hline $\begin{array}{l}\text { Meetings observés } \\
\text { (ordre } \\
\text { chronologique) }\end{array}$ & $\begin{array}{l}\text { Nombre de } \\
\text { participants } \\
(\text { selon nos propres } \\
{\text { estimations })^{10}}\end{array}$ & $\begin{array}{l}\text { Date } \\
\text { (heure) }\end{array}$ & Salle \\
\hline $\begin{array}{l}\text { Marine Le Pen } \\
\text { Front National }\end{array}$ & Env. 1500 & $\begin{array}{l}\text { Dimanche } 5 \\
\text { février } \\
(15 \mathrm{~h} 30)\end{array}$ & Hall 7 Parc des expositions \\
\hline $\begin{array}{l}\text { François Bayrou } \\
\text { Mouvement Démocrate } \\
\text { (MoDem) }\end{array}$ & Env. 2000 & $\begin{array}{l}\text { Samedi } \quad 10 \\
\text { mars } \\
(18 \mathrm{~h} 30)\end{array}$ & La Halle aux grains \\
\hline $\begin{array}{l}\text { Nathalie Artaud } \\
\text { Lutte ouvrière }\end{array}$ & Env. 250 & $\begin{array}{l}\text { Vendredi } 30 \\
\text { mars } \\
(20 \mathrm{~h} 30)\end{array}$ & Salle des Allées de Barcelone \\
\hline $\begin{array}{l}\text { Jean-Luc Mélenchon } \\
\text { Front de Gauche }\end{array}$ & Env. 60000 & $\begin{array}{lll}\text { Jeudi } & 5 & \text { avril } \\
(19 h) & \end{array}$ & $\begin{array}{l}\text { Place du Capitole (+ rues } \\
\text { adjacentes et place Wilson) }\end{array}$ \\
\hline $\begin{array}{l}\text { Philippe Poutou } \\
\text { Nouveau } \\
\text { Anticapitaliste }\end{array}$ & Env. 1200 & $\begin{array}{l}\text { Mardi } 17 \text { avril } \\
(20 \mathrm{~h} 30)\end{array}$ & Salle Mermoz \\
\hline
\end{tabular}




\begin{tabular}{|l|l|l|l|}
\hline $\begin{array}{l}\text { Nicolas Sarkozy } \\
\text { Union pour un Env. 6000 } \\
\text { Mouvement Populaire }\end{array}$ & $\begin{array}{l}\text { Dimanche 29 } \\
\text { avril } \\
(15 \mathrm{~h} 30)\end{array}$ & Hall 5 Parc des expositions \\
\hline $\begin{array}{l}\text { François Hollande } \\
\text { Parti Socialiste }\end{array}$ & Env. 60000 & $\begin{array}{l}\text { Jeudi 3 mai } \\
(18 \mathrm{~h} 30)\end{array}$ & $\begin{array}{l}\text { Place du Capitole (+ rues } \\
\text { adjacentes et place Wilson) }\end{array}$ \\
\hline
\end{tabular}

Source : collectif SPEL Meetings toulousains, 12/12/2013.

\section{Accueillir les participants et sécuriser le public : une logique disciplinaire}

7 En mars 2012, François Bayrou tient son meeting à la Halle aux grains, une salle de spectacle de Toulouse (figure 1).

Figure 1. Meeting de François Bayrou, La Halle aux grains, 10/03/2012 (18h30)

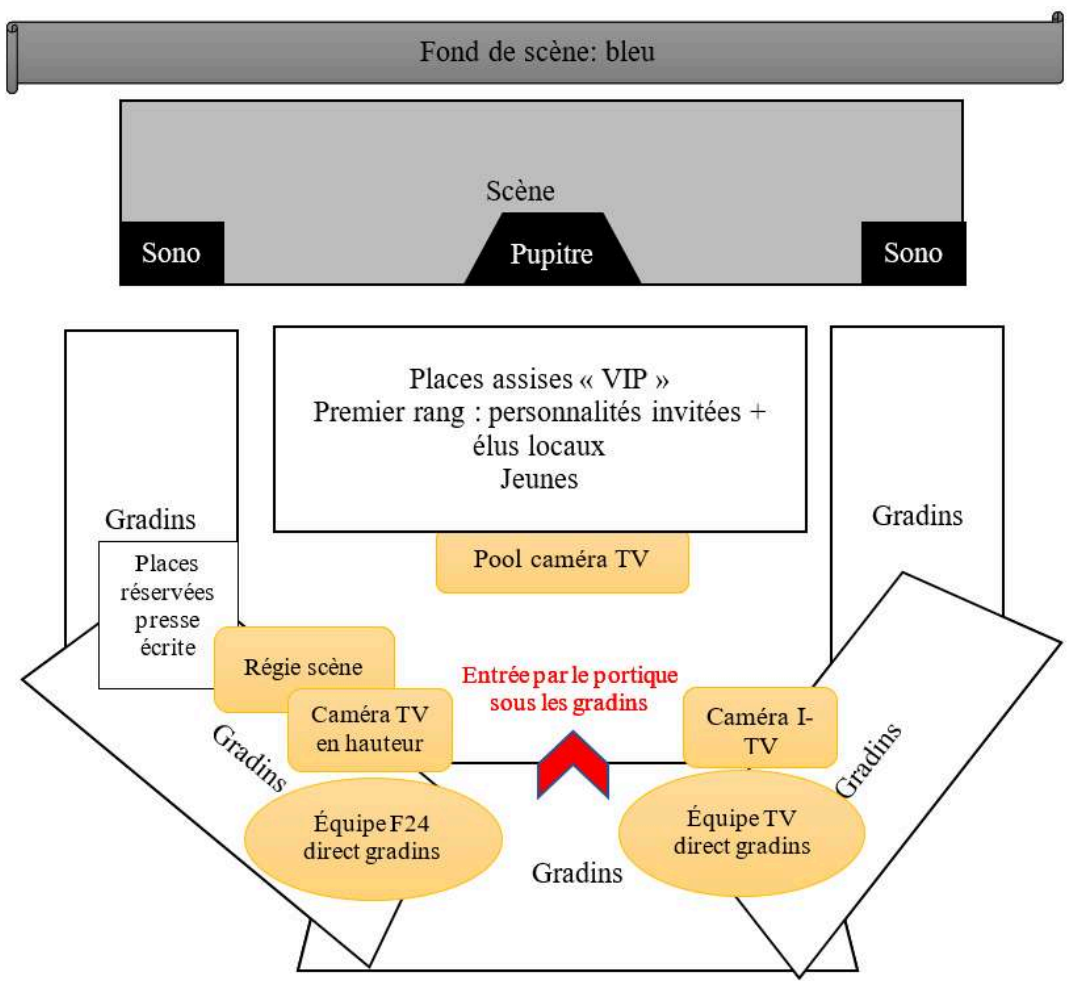

Source : collectif SPEL Meeting toulousain, 12/12/2013.

Cette salle située en centre-ville possède une grande capacité d'accueil (environ 2000 places assises). Majoritairement utilisée pour des concerts de musique classique et des spectacles, elle est située à proximité des quartiers les plus riches du centre-ville. Son accès est, à première vue au moins, ouvert et simple, à proximité pour les habitants du centre et bien desservie par les transports en commun. Toutefois, les places de parking dans le centre-ville de Toulouse étant majoritairement payantes, le choix de cette salle constitue un premier filtre quant au public attendu. L'organisation spatiale de la salle en est un second : s'appuyant sur l'existant (la hiérarchie propre aux salles de spectacle 
entre les places les plus prisées et les plus chères au-devant de la scène et les gradins), l'agencement de la salle vise à accueillir et accompagner un public d'habitués dans un espace reproduisant les hiérarchies défendues au sein du parti. En effet, comme lorsqu'ils assistent à un concert, les participants ne peuvent pas réellement aller où bon leur semble. Dès l'entrée, le public est réparti et organisé dans les différents gradins par des militants et des membres de l'équipe nationale de campagne bien visibles, qui l'accueillent sur un registre s'apparentant à celui des hôtesses des congrès commerciaux, auxquelles ils ressemblent jusque dans leurs tenues. Les hommes comme les femmes portent des tailleurs noirs très classiques avec des chemises blanches. Cette façon de représenter l'ouverture et l'accessibilité au public par la mise à disposition d'un personnel d'accueil identifiable est donc très située socialement et s'adresse à une population issue des classes moyennes et aisées (Fretel, 2004) aux corps (auto-) disciplinés (Bourdieu, 1979 ; Régnier, Masullo, 2009 ; Comby, Grossetête, 2012).

Des rangées de chaises « VIP », réservées aux personnalités du parti (élus, responsables politiques, personnalités invitées et quelques jeunes militants $d u$ parti) ont été installées sur le parterre en face de la scène quand les spectateurs lambda ont été placés dans les gradins latéraux. Ceux placés sur les rangs du haut ne voient même pas vraiment la scène et suivent ce qu'il s'y passe en regardant les images diffusées sur de petits écrans plats au-dessus de leurs têtes. La distribution spatiale évoque ainsi une société très hiérarchisée.

Sur un autre pan de l'échiquier politique, le meeting de Jean-Luc Mélenchon (Front de gauche), sous ses apparences de grande ouverture et de liberté puisqu'il s'agit d'un meeting en plein air sur une place publique de centre-ville, laisse transparaître lui aussi une forme de tri et de conduite des conduites des participants (figure 2). La place du Capitole est un lieu de rassemblement ouvert pouvant accueillir des milliers de personnes. 
Figure 2. Meeting de Jean-Luc Mélenchon, Place du Capitole, 05/04/2012 (19h)
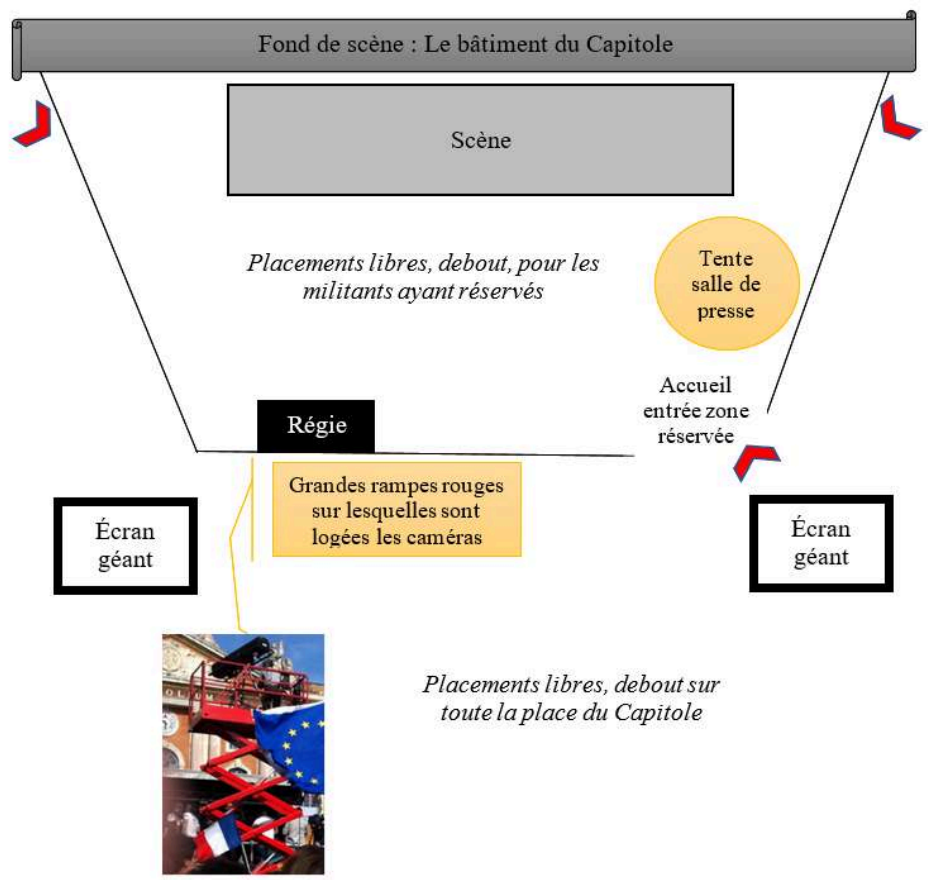

Placements libres, debout sur

toute la place du Capitole

Source : collectif SPEL Meeting toulousain, 12/12/2013

11 La présence d'un « carré VIP » (pour "Very Important Person », en l'occurrence pour les militants actifs du parti, les élus ou dignitaires étant à cette période encore peu nombreux) relève de stratégies de représentation médiatique et d'une forme de rétribution militante (Gaxie, 1977). En effet, ce carré VIP séparé par des barrières avec une entrée surveillée semble surtout avoir une fonction de médiatisation : y sont placés les militants porteurs des signes d'identification au parti qui vont permettre la production d'images contrôlées d'un meeting animé où les drapeaux volent au vent, donnant à voir une « marée rouge ».

Les militants du carré VIP du Front de gauche sont ainsi les seuls à composer le public « direct » du candidat. La rampe médiatique située derrière eux barre littéralement la visibilité de la scène (figure 2). En dehors du carré VIP, les spectateurs n'ont donc pas d'autre choix que de regarder les écrans géants: cette vision en synchronie mais médiatisée crée de fait une division nette du public. Aussi, si le meeting est aisément accessible à tous, le public mis en représentation, celui qui apparaitra à la télévision et qui est le plus proche du candidat, est pour sa part sélectionné et organisé. La représentation symbolique de la société ainsi évoquée dans la distribution spatiale met en exergue deux groupes : les membres du parti, sélectionnés et choisis pour intégrer le carré VIP, et les autres.

13 Par ces dispositifs différents, le Modem et le Front de Gauche mettent en valeur dans leurs parterres VIP deux registres de légitimités, élective et notabiliaire pour l'un, populaire pour l'autre (Gaxie, 2004). Pour autant il reste que ces meetings sont formellement ouverts et accessibles à tous. Pour d'autres groupes au contraire - Front National (FN), UMP - il s'agit de choisir le public et de l'encadrer strictement afin d'en assurer la sécurité. La discipline recherchée n'est plus adressée à un large public mais 
au groupe partisan qu'il faut protéger d'une menace extérieure réelle ou supposée mais aussi d'une menace intérieure, ici aussi réelle ou supposée, par un tri sélectif des participants et un dispositif visible de service d'ordre se voulant dissuasif et relevant d'une vision de l'ordre public disciplinaire.

Dans le cas du meeting du FN, le public visé est avant tout un public de convaincus. Non seulement les organisateurs ne s'attendent pas à accueillir des curieux, mais ils les dissuadent de venir en mettant en place une entrée payante ( 5 euros) qui permet d'« éviter les hostiles $»^{11}$. Selon une responsable du service d'ordre, celui-ci est composé d'environ soixante membres à l'extérieur ${ }^{12}$ et d'une quarantaine de membres à l'intérieur de la salle ${ }^{13}$. Cette répartition invite à relativiser l'ampleur de la menace extérieure dans ce dispositif: si les opposants extérieurs sont perçus comme les adversaires directs, le public est lui aussi perçu comme une menace potentielle. En effet, il pourrait d'une part être infiltré par des personnes extérieures non désirées, comme le rappelle ce membre du service d'ordre, ancien militaire de Djibouti qui lance à la cantonade lors de notre passage «ceux qui viennent foutre la merde, qui sont contre le FN... on fait fouille intégrale... $»^{14}$. D'autre part, comme l'indique un responsable, ce dispositif vise aussi à se protéger des sympathisants eux-mêmes, parfois considérés comme " très énervés » ${ }^{15}$.

L'exemple du meeting du FN incarne à son paroxysme l'idée que l'organisation du public fait pleinement partie de la vision politique proposée par le parti en représentation. Au-delà de ce qui est dit, l'expérience physique du meeting est déjà un message.

Le meeting de Nicolas Sarkozy se déroule un après-midi d'avril au Parc des Expositions, un lieu en périphérie de Toulouse qui n'est pas desservi par les transports en commun. Ce choix géographique est en partie imputable à la décision tardive de l'équipe de campagne d'organiser un meeting à Toulouse. Cette ville est alors considérée par les commentateurs politiques comme très marquée à gauche et très investie et disputée par le PS et le Front de Gauche ${ }^{16}$. Pour autant, nos observations nous conduisent à avancer un autre élément explicatif à ce choix d'une salle relativement excentrée : la volonté de mieux gérer voire de limiter le public. Une discussion informelle avec un agent du service d'ordre met en effet en avant la crainte des organisateurs de ne pas avoir assez de monde ou d'avoir des perturbateurs. Cette double inquiétude les a ainsi conduits, selon lui, à ne pas faciliter l'accès depuis le centre-ville et à organiser à l'intérieur de la salle des espaces très compartimentés avec de grandes travées infranchissables. L'organisation spatiale de la salle était donc très stricte pour maitriser un public en grande partie invité. Ce sont d'ailleurs les images qu'en proposent les équipes professionnelles engagées par le candidat, diffusées sur les petits écrans du hall d'entrée et qui seront ensuite gracieusement offertes aux différents médias n'ayant pas pu tourner leurs propres images (Haffaf, Nollet, 2013). Les plans filmés de la salle donnent à voir un public enthousiaste et discipliné qui rend assez mal compte de ce qui se trame dans le hall ni même dans la salle. 
Figure 3. Meeting de Nicolas Sarkozy, Parc des expositions de Toulouse, 29/04/2012 (15h30)

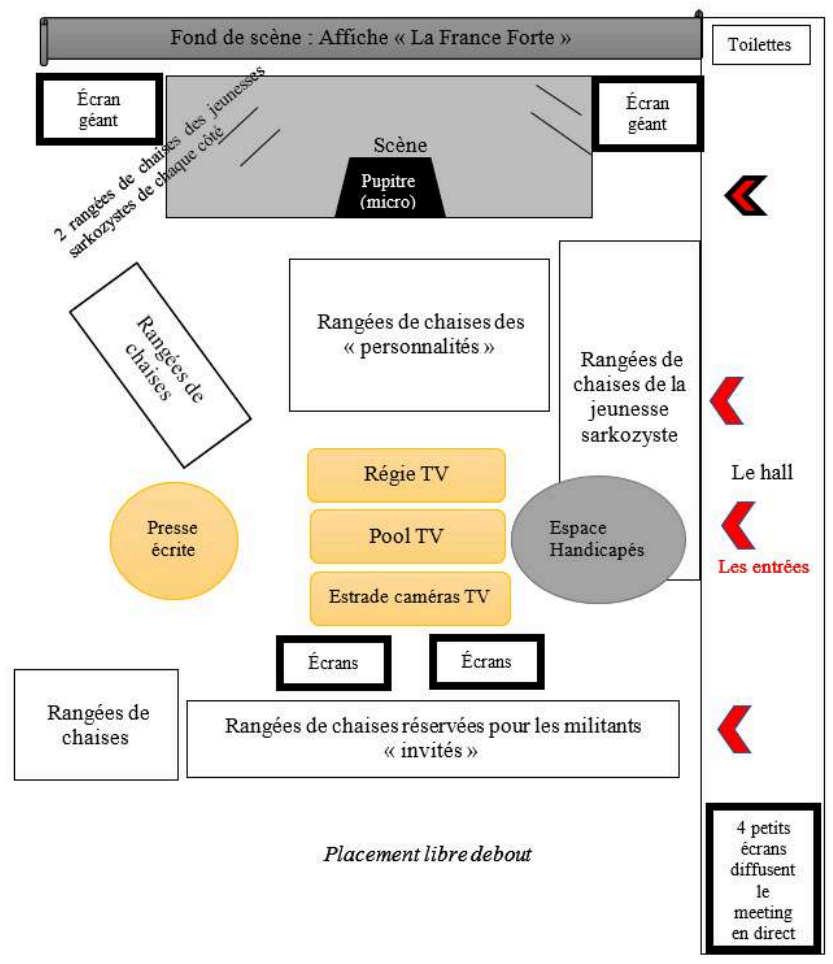

Source : collectif SPEL Meeting toulousain, 12/12/2013.

17 L'affluence de visiteurs venus de façon autonome perturbe ce contingentement. À l'extérieur, le parking est rapidement complet et certaines personnes restent même bloquées dans leurs voitures ne trouvant pas de place pour se garer ${ }^{17}$. Alors que les militants arrivant en bus sont directement conduits à l'intérieur par des entrées inaccessibles aux autres (porte de derrière), les spectateurs venus par leurs propres moyens, trop nombreux au regard de l'espace destiné à les accueillir, sont contraints de s'agglutiner dans le hall devant les portes d'entrée. Ainsi maintenus à l'extérieur de la salle, ils sont réduits à suivre sur de petits écrans (figure 3) les discours retransmis en direct, dans la chaleur et la cohue. Certains préfèrent finalement discuter à l'extérieur : « on tient pas là-dedans, c'est n'importe quoi... ».

À l'intérieur, l'organisation stricte de la salle par un découpage en espaces isolés les uns des autres n'apparaît ainsi pas uniquement comme une préoccupation de sécurité ${ }^{18}$. Le public du meeting de l'UMP est constitué de ceux ayant réussi à entrer, soit parce qu'ils étaient prévus (militants, invités, journalistes) soit parce qu'ils étaient présents suffisamment tôt pour pénétrer la salle avant les autres. Ce public est physiquement et symboliquement séparé en divers groupes (les «jeunes», les "invités», les "personnalités ", la presse, les personnes en situation de handicap moteur) et renvoie l'image d'une société fortement compartimentée en groupes distincts, un espace public idéal divisé et contrôlé. Ces différents groupes entrent par une porte dédiée et sont répartis par les organisateurs selon un plan de salle bien défini à l'avance. Cette organisation spatiale renvoie au discours politique de l'UMP d'une société hiérarchisée et ordonnée.

19 Ce meeting, dont le dispositif apparait au premier abord comme le plus structuré et organisé, se solde finalement par un important désordre et le mécontentement d'une 
grande partie du public. L'insatisfaction ne se limite pas aux spectateurs "exclus» puisqu'à la fin du meeting les militants et sympathisants invités se plaignent euxmêmes d'une salle où « il faisait extrêmement chaud » sans qu'il n'y ait pour autant de lieu prévu pour se ravitailler en eau ${ }^{19}$. Cette chaleur a ainsi provoqué des évanouissements de personnes dans la salle comme dans le hall, qui sont alors portées au-dessus de la foule par les pompiers ou des membres du service d'ordre. Parmi les participants venus en dehors de toute organisation du parti et maintenus à l'extérieur de la salle, l'exaspération est telle lorsque le meeting s'achève que certains souhaitent initier une manifestation spontanée jusqu'à la mairie pour se plaindre d'une "organisation inacceptable». Cette dernière, résultant de la sous-estimation de l'enthousiasme produit par la candidature du président sortant et par un rapport particulièrement inquiet au public, ne pouvait, selon ces participants évincés, qu'être une volonté de "sabotage de la mairie socialiste ${ }^{20}$.

L'organisation spatiale, le placement des corps dans l'espace, les modalités d'accueil et de filtrage des participants des meetings sont donc plus qu'une contingence matérielle. Cela participe de la représentation politique au sens de mise en scène du monde social et de ses divisions, proposée par les groupes partisans.

\section{De la visibilité des services d'ordre : contrôler « l'espace public idéal »}

21 Les enjeux disciplinaires et de maintien de l'ordre sont induits par le dispositif spatial et incorporés par les participants. Alors que dans certains meetings la présence du service d'ordre est invisibilisée et relayée par un dispositif permettant à tout un chacun de rester à la place qui lui a été suggérée (comme c'est le cas pour le meeting de François Bayrou décrit plus haut), dans d'autres meetings, le service d'ordre très visible apparaît central dans l'organisation. Cette dernière est alors essentiellement tournée vers la gestion de la menace, qu'elle soit intérieure ou extérieure et que le public soit appréhendé comme une masse potentiellement hostile ou comme un groupe à protéger de la menace des opposants (dans le cas du Front National par exemple). Ces différences dans la construction de l'ordre du meeting renvoient à la fois aux histoires militantes propres aux partis, aux conjonctures dans lesquelles se déroule le meeting mais aussi aux positionnements politiques de chaque parti et donc à leur conception de l'espace public et de l'ordre social.

Concernant la sécurité du candidat en représentation, de manière générale, la distinction entre protection rapprochée des personnalités et sécurisation de l'évènement est particulièrement nette mais cette protection rapprochée est plus ou moins visible. Les partis les plus puissants dans le champ politique ont pour la plupart recours aux services du SPHP (Service de protection des hautes personnalités, un service de police) pour la protection de leur candidat. Marine Le Pen bénéficie ainsi de deux agents du SPHP que nous n'avons pas vus et François Bayrou comme Éva Joly d'un seul agent. Au meeting du Parti Socialiste, les services de protection rapprochée sont habillés en civil, dans cette forme toute particulière de tenue qui permet néanmoins d'identifier celui qui la porte comme étant policier ou agent de sécurité. Lors du bain de foule du candidat, ils sont doublés d'un service d'ordre composé de militants du Parti Socialiste relativement massifs, vêtus en civil et arborant un badge "SO équipe de campagne $»^{21}$. Mais pour le meeting de l'UMP ce sont les services de police qui sont 
directement chargés de la protection de Nicolas Sarkozy, en tant que président de la République en campagne pour sa réélection.

Dans le cas des meetings se déroulant sur une place publique, à la gestion de l'ordre interne du meeting et de la sécurité du candidat s'ajoute la nécessité d'une prise en compte de l'ordre public (sécurisation du périmètre du meeting, protection des biens publiques, gestion des foules). Pour les équipes organisatrices, cela suppose de demander des autorisations préfectorales et de mobiliser des militants sur ce poste. $\mathrm{Au}$ Front de gauche, l'organisation de la sécurité de l'évènement a donné lieu à des négociations internes au groupe politique, notamment sur l'appropriation de l'espace public (encadré 2). Cette prise en charge de la sécurité de l'événement par les militants non spécialisés participe à l'invisibilisation du dispositif de sécurité pour privilégier la convivialité et la protection de tous les sympathisants: "Nous sommes là pour protéger les gens et il est donc très important d'être aimable et poli! $\aleph^{22}$. Le service d'ordre revêt ainsi la forme d'un service d'accueil qui oriente et protège le public en tant que foule avec ses mouvements potentiellement dangereux et ses imprévisibilités. Il s'agit d'une évolution que l'on retrouve ailleurs, notamment dans le rôle des services d'ordre des syndicats dans les manifestations (Sommier, 1993).

\section{Encadré 2. Un service d'ordre protecteur}

Carnet de terrain ${ }^{23}$, réunion de préparation avec les volontaires du service d'ordre du Parti de Gauche le 5 avril 2012 à $15 h$.

Ce jour-là, à $15 \mathrm{~h}$ nous nous rendons à la réunion du service d'ordre en suivant les gens qui ont des autocollants. Cela n'est pas difficile d'entrer dans la salle où la réunion se déroule, personne ne contrôle vraiment les entrées et il suffit d'avoir l'air de savoir où l'on va. Là nous pouvons constater que l'organisation est très fragile.

Il semble que les conflits sur les autorisations d'utilisation de certains espaces avec la mairie entrainent un décalage dans l'organisation de la réunion. Les organisateurs sont pris de court et réagissent au pied levé. Les écrans qui ont été installés sur la place Wilson viennent d'être interdits et une partie importante des responsables d'équipes du service d'ordre qui devaient assister à cette réunion sont toujours sur la place. C'est le bras de fer avec la mairie.

Dans la salle il y a des gens qui viennent de toute la région. C'est le Parti de Gauche $^{24}(\mathrm{PG})$ qui est responsable de la sécurité des meetings de la campagne présidentielle et comme il compte assez peu de militants et il a fait appel aux volontaires issus de groupes militants très variables. Des groupes locaux et sympathisants ont proposé des gens pour participer à la sécurité. Dans cette réunion il y a environ $40 \%$ de femmes et la moyenne d'âge se situe autour de 45-50 ans. Il y a une dizaine de groupes auxquels ont été attribués des noms révolutionnaires «Babeuf 1/ Babeuf 2 » ou « Gracchus 1 ». C'est donc un groupe très disparate de femmes, d'hommes, de très jeunes, d'assez vieux qui est rassemblée dans cette salle pour organiser la sécurité du meeting. À cette grande hétérogénéité s'ajoute l'approximation. En effet, l'homme qui gère les groupes et leurs placements ne connait pas Toulouse et indique de façon très vague des lieux précis à des participants pour une grande part non Toulousains. Il y a des 
flottements et il se fait sérieusement taquiner et même applaudir quand ses indications sont vraiment trop confuses. L'ambiance reste très joyeuse et festive. Il indique qu'en faisant les équipes ils ont essayé dans la mesure du possible de laisser les gens qui venaient du même département ensemble. La présence sur deux sites, la place du capitole et la place Wilson, ne facilite pas l'organisation.

Le Front de gauche affiche ainsi sa volonté de mettre en scène le peuple dans sa diversité jusque dans le service d'ordre dont la mission est clairement de gérer les mouvements de foule afin d'assurer la sécurité du public. Il doit faire en sorte qu'il n'y ait pas de gens piétinés ou de personnes qui s'évanouissent, et de ménager des couloirs sanitaires. Il s'agit, comme les noms des équipes l'évoquent, du « peuple en marche vers une révolution dans les urnes ${ }^{25} »$. En ce sens, protéger le public, c'est au moins autant protéger le parti en représentation que défendre le dispositif politique légitime: le vote.

Dans un autre registre, lors du meeting de François Bayrou, le service d'ordre est lui aussi quasiment invisible en dehors des gardes du corps du candidat. La sécurité du meeting a été déléguée à des prestataires de services habitués à endosser cette responsabilité sur des évènements d'autre nature - sportifs, culturels ${ }^{26}$.

À l'inverse, dans certains meetings comme celui de Lutte Ouvrière présenté en ouverture d'article, les services d'ordre sont délibérément très visibles car cette exposition de l'équipe de maintien de l'ordre est doublement stratégique. D'un côté, elle vise une "disciplinarisation" des conduites (Foucault, 1975), de l'autre elle objective une représentation ordonnée, contrôlée, hiérarchisée de l'espace public idéal.

Concernant la « disciplinarisation » des conduites, l'omniprésence des agents tend ainsi à produire sur le public un effet de surveillance permanente, à la fois par ce qu'elle montre et par ce qu'elle ne montre pas. La "visibilité est un piège» disait Michel Foucault, le nombre d'agents (a priori) visibles rend (pratiquement) impossible la perception de l'activité de chacun et, surtout, il laisse croire à la présence d'agents invisibles, d'où la dimension "panoptique» du dispositif. Ce dernier a pour effet majeur de «faire que la surveillance soit constante dans ses effets, même si elle est discontinue dans son action » (Foucault, ${ }_{1975: 234)}$. Par exemple, durant le meeting du FN le service d'ordre est déployé, visible et tendu, essaimé dans tout l'espace. Les agents ne regardent pas la scène mais seulement la salle, le moindre mouvement brusque attire leur regard et ils ont des oreillettes pour communiquer entre eux. Ces agents quadrillent la salle : ils sont environ dix de chaque côté, huit dans la rangée du milieu et une bonne quinzaine au fond (voir la disposition de la salle, figure 4). À cela s'ajoute des agents volants ainsi qu'une dizaine de membres du service d'ordre stationnés devant la scène. Ici, la clôture de l'espace du meeting par ce dispositif de sécurité renvoie directement à la crainte des débordements, à la représentation du désordre exogène potentiellement intrusif, d'où un dispositif de sécurité tout entier tourné vers la dissuasion et le contrôle du public. 
Figure 4. Meeting de Marine Le Pen, Parc des expositions de Toulouse, 05/05/2012 (15h30)

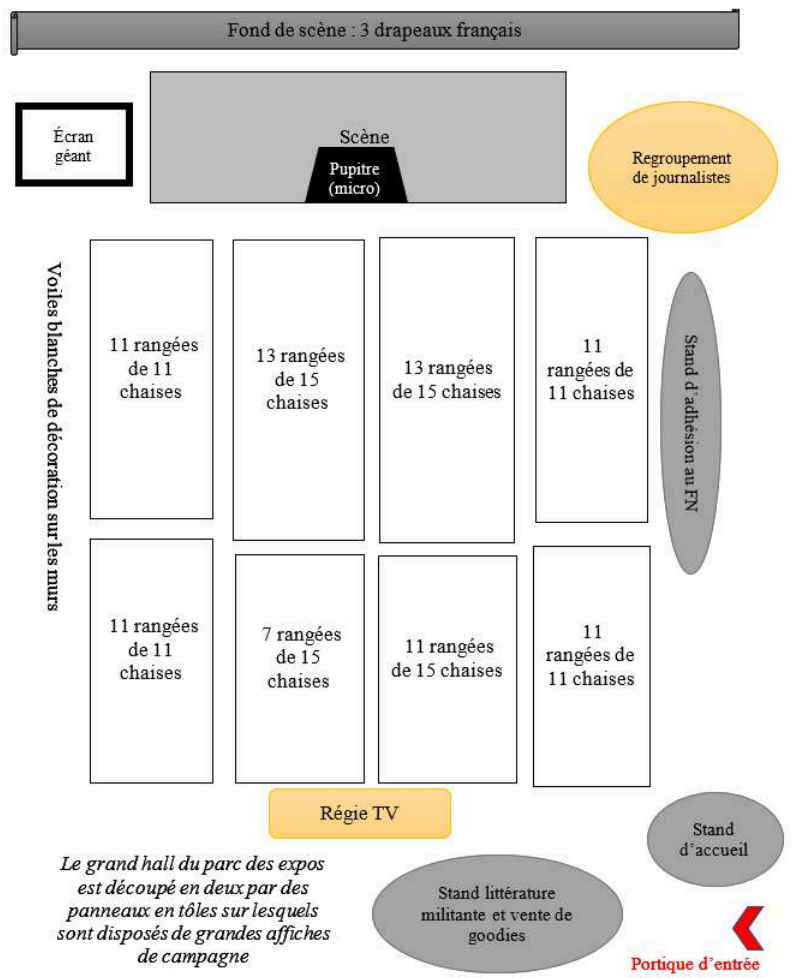

Source : collectif SPEL Meeting toulousain, 12/12/2013.

La force de certains collectifs partisans passe par la mise en ordre minutieuse, disciplinée voire militaire (comme l'activité professionnelle de certains membres du service d'ordre du FN le rappelle) des militants comme des sympathisants. Ainsi, au Front National, les conditions d'accueil paraissent d'autant plus fermées que le service d'ordre est très présent, visible et actif dès l'entrée. Des portiques détecteurs de métaux sont installés comme dans un aéroport pour accueillir chaque visiteur qui doit vider le contenu de ses poches dans un petit panier avant de passer. C'est le seul meeting où un tel dispositif a été observé. Le meeting de Nicolas Sarkozy qui offre quant à lui le plus gros dispositif de sécurité (notamment parce que le service d'ordre et le service de protection rapprochée du candidat se doublent d'un déploiement des forces de l'ordre impressionnant), n'en est pas moins celui qui présente le plus de débordements.

Aussi, qu'elles soient visibles ou invisibles, dissuasives ou accueillantes, les différentes modalités de filtrage des participants, d'organisation du public, de répartition et de contrôle des corps dans l'espace participent d'une même volonté de «disciplinarisation» des conduites par l'organisation spatiale et sociale du meeting. Les services d'ordres, privés ou largement constitués d'agents publics, professionnels ou pas, sont des indicateurs éloquents de la façon dont le groupe partisan en représentation conçoit les forces de l'ordre et leur place dans l'espace public. 


\section{Mises en scène et en ordre du corps social dans l'espace : maintenir le public du meeting à bonne distance}

Si le meeting est un invariant du «faire campagne » (Baamara, Floderer, Poirier, 2016; Desrumaux, Lefebvre, 2016), les façons variables de spatialiser cet évènement incarnent différentes manières de concevoir la représentation politique ainsi que le rôle des profanes et des professionnels, dont le candidat plus ou moins présidentiable. Envisager le meeting en gardant à l'esprit la matérialité des relations de pouvoir permet de rappeler qu'il s'agit bien de l'incarnation physique du « consensus qui s'est établi sur l'organisation politique de la société qui contribue à renforcer la tendance anthropologique à la sacralisation du pouvoir » (Gaxie, 2004). Le meeting comme rituels de séduction et d'institution (Bourdieu, 1982) en campagne électorale a ainsi pour vocation, comme à l'église, de stimuler et simuler la ferveur du peuple en même temps que la reconnaissance de la délégation et donc des délégués en particulier. Ce processus de séparation politique passe, dans chaque meeting, par la représentation, une et multiple, d'un corps social ordonné dans l'espace et séparé des professionnels (militants aguerris, VIP, organisateurs, élus, notables du parti et bien sûr le candidat). Cette distribution des rôles est donc aussi une distribution spatiale de l'expression politique légitime (Baloge, 2016). L'événement en lui-même constitue une mise en ordre et en représentation à la fois du jeu électoral mais aussi de la place de chacun dans celui-ci. Chaque mise en scène du rituel renvoie à des modes de contrôles qui se déploient dans l'espace, soit une "gouvernementalité spatiale» qui s'appuie sur la gestion des espaces plutôt que sur les personnes (Merry, 2001). Cette orientation des comportements par des formes d'incitation s'éloigne de l'imposition disciplinaire sans pour autant se traduire par un effacement des hiérarchies politiques et sociales, ni par l'absence d'encadrement des participants.

31 Aussi, dans un premier temps, il s'agit de rendre compte d'une forme de gouvernementalité par la distance en revenant sur l'ensemble des éléments matériels, des dispositifs visuels et sonores plus ou moins élaborés visant à médier et à rendre spectaculaire la relation à la personnalité politique et au-delà à la politique. Dans un second temps, l'objectif est de montrer que dans certains groupes politiques très nettement positionnés à gauche, l'organisation du meeting tente de dépasser ou de minimiser la personnalisation et l'individualisation du pouvoir afin d'incarner, à travers une forme de gouvernementalité par la proximité, une revendication de politique pour et par tous sans pour autant aller jusqu'à transgresser totalement l'essence du rite fondée sur la séparation entre professionnels et profanes.

\section{Personnalisation et médiation : une gouvernementalité par la distance}

Marc Abélès (2010) l'a montré, le meeting comme tout rituel politique est un mélange entre l'émotion et l'artifice, l'exception et la banalité, la proximité et l'éloignement. La scène est investie de différents symboles qui sont censés créer une ambiance, un espace de significations, et, au-delà, guider les corps et l'attention vers ce qui est conçu comme le cœur de l'événement. Se déploient ainsi différents supports qui accompagnent la présence d'un candidat et qui appuient le sens spécifique de chaque meeting: codes 
couleurs, affichages, vidéos, drapeaux, banderoles, etc. Chacun de ces éléments a une fonction pratique et idéologique. Certains rythment le temps du meeting, comme l'agitation des drapeaux. D'autres permettent de se connaître, de se reconnaitre et d'apparaître sur les écrans (banderoles régionales, codes couleurs, pancartes). Tous participent à la normalisation des comportements des membres du public pour susciter une adhésion à l'événement et au-delà à l'ordre politique que constitue la délégation et la représentation politique par des professionnels.

Cela participe d'une "gouvernementalité » des conduites dans le sens où maintenir l'ordre du meeting c'est aussi participer à son bon déroulement, susciter l'adhésion et rallier à sa cause le plus grand nombre. Il y a donc un encadrement de l'ambiance et de l'implication du public: par le cadrage visuel, avec notamment les médias ou par le cadrage sonore, avec notamment la présence ou l'absence de chauffeurs de salles qui rythment les applaudissements, la musique d'ambiance de l'avant meeting, de l'entrée du candidat et de sa sortie, mais aussi avec les activités annexes au meeting. Sur ce dernier point l'accès à des camions de restauration, ou des restaurants, l'intervention de groupes de musiques sur scène avant ou après le meeting, et la présence d'alcool (en vente sur le lieu de l'événement ou apporté par les participants) sont, selon nos observations, plutôt une caractéristique des meetings de gauche, ce qui apparaît comme volonté de libération des corps et de cohésion. Cette valorisation de la dimension festive et ludique est très située politiquement, les partis de droite jouant moins sur ce registre, hormis les soirs de victoire.

Plusieurs signes permettent de rapprocher les meetings politiques contemporains des partis électoralement les plus puissants du dispositif du spectacle ou du concert (Cossart, 2010). Tout d'abord, le candidat délivrant son discours seul à la tribune est au centre de l'attention. Le public vient voir cette personnalité, dont l'intervention, comme dans les concerts, est précédée par une première partie. Celle-ci doit permettre à la fois aux orateurs de s'entrainer à l'exercice qu'est la prise de parole en meeting, mais elle sert aussi à " chauffer la salle " pour rendre l'entrée du candidat encore plus spectaculaire. Cette théâtralisation des entrées et sorties du candidat est un phénomène qui date des années 1930 (ibid. : 267). Le fait de cacher le candidat avant le meeting, de multiplier les interventions avant son arrivée sur scène, fait partie d'une stratégie générale de personnalisation. En cela, l'intervention de Marine Le Pen se rapproche du one-woman-show, lorsqu'elle parcourt la scène de part et d'autre en faisant de grands gestes, mimant parfois certaines parties de son discours ${ }^{27}$ délivré en arpentant la scène grâce à un micro-cravate ${ }^{28}$. Pour autant elle ne se prête pas vraiment au rituel du bain de foule (voir l'analyse de ce rituel ci-après) et reste la plupart du temps seule en scène hormis pour le chant de la Marseillaise à la fin. Cette façon de représenter le leader seul face au peuple dépasse le choix de mise en scène pour incarner un message politique.

Dans le cas du meeting de François Hollande sur la Place du Capitole, la mise en scène de l'événements présente de nombreux points communs avec celle d'un spectacle musical grand public, de type concert de rock (figure 5). 


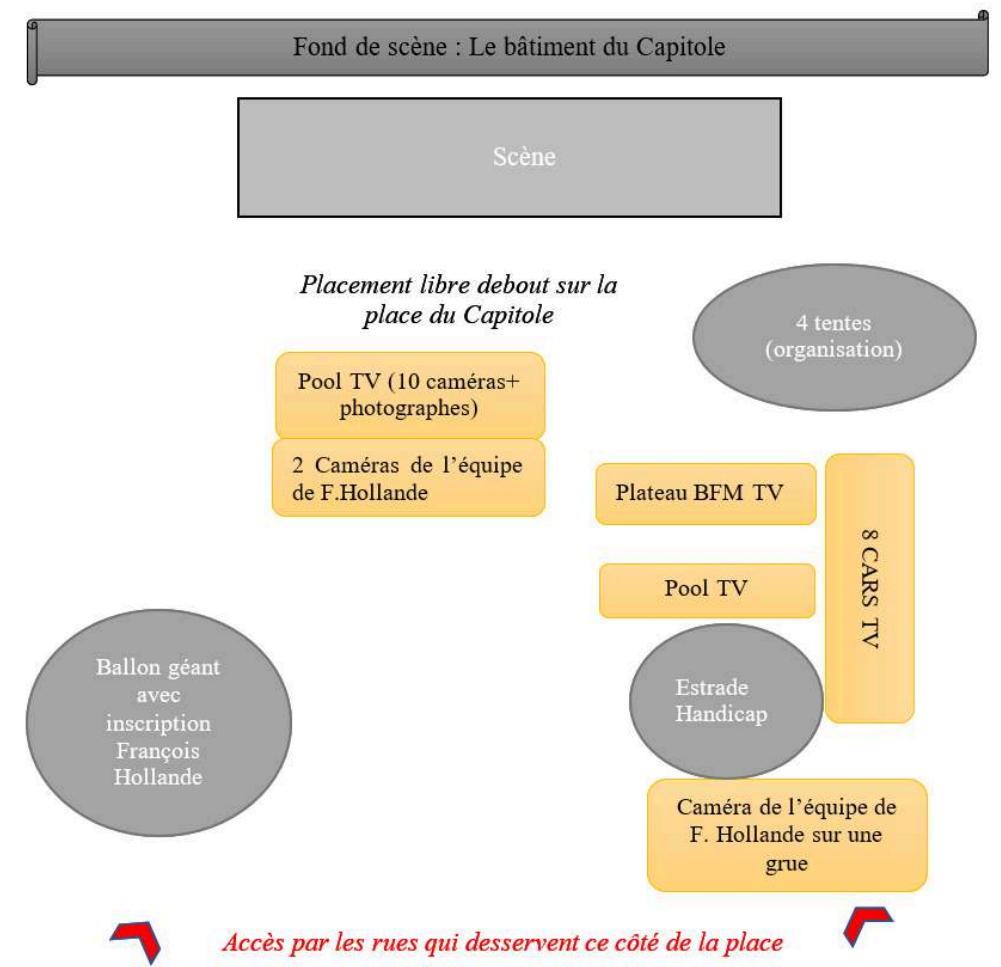

Source : collectif SPEL Meeting toulousain, 12/12/2013.

Après une première partie consacrée à des groupes de musiques (Les Grandes Bouches, Michel Fugain et Axel Bauer) ${ }^{29}$, des membres du parti animent la soirée, puis passent la parole à plusieurs intervenants avant d'annoncer enfin la venue de la «star de la soirée ", qui arrive elle-même en déclarant haut dans le micro "bonsoir Toulouse! ». Pendant toute cette première partie plusieurs personnes peuvent être sur scène, mais lorsque François Hollande fait son discours, il est seul derrière son pupitre et constitue l'unique objet d'attention. Tout est fait pour que les regards ne soient posés que sur lui directement ou à travers les écrans. À la fin, il prolonge l'ambiance musicale en diffusant successivement la Marseillaise puis sa musique de campagne. Cela lui permet de rester un peu plus longtemps sur scène, d'y agiter un drapeau français que lui donne un militant dans le public, puis de prendre un bain de foule, serrant les mains des uns, embrassant les autres, sur des musiques populaires comme celles du groupe Téléphone. Ce moment du bain de foule est un rituel attendu, qui se déroule, selon les meetings, à l'entrée ou la sortie de scène, ou parfois même les deux. Cela permet de représenter la liesse populaire et ce sont des images très reprises dans les médias (Haffaf, Nollet, 2013 ; Sécail, 2016). Le bain de foule est donc un élément important du dispositif meeting parce qu'il rappelle en pratique l'enjeu de délégation politique et le coup de force symbolique qui permet à un individu candidat de représenter son groupe politique et au-delà, le peuple qui le soutient (Bourdieu, 1981). Le bain de foule constitue aussi une forme de rétribution militante, notamment pour les jeunes militants qui sont en général sur le parcours du candidat. Il n'est pas propre aux meetings de gauche mais ce moment où les contacts sont très orchestrés est en général réservé aux présidentiables, c'est-à-dire à ceux que les sondages placent dans les trois voire quatre premières places (dans cette campagne Nicolas Sarkozy, François Hollande 
et Marine Le Pen). Ces derniers le pratiquent régulièrement mais de façon assez différente, notamment à Toulouse. On l'a vu Marine Le Pen ne traverse pas la salle, elle ne se « mêle » qu'au premier rang à sa sortie de scène. Nicolas Sarkozy suit un parcours strict et assez rapide dans la salle à son entrée et sa sortie, en particulier à proximité du carré des jeunes militants et des VIP. Seul François Hollande fait durer ce moment et met son service de protection et le service d'ordre à l'épreuve de la gestion prolongée de cette proximité. Cette dernière (tout comme le dispositif de médiation et d'encadrement visuel et sonore) ont paradoxalement pour effet de mettre le candidat à distance, de le présenter comme un personnage hors du commun pouvant endosser le rôle de président (Lacroix et Lagroye 1992).

Ce jeu de proximité et de distance avec le candidat semble renforcé dans le cas des meetings extérieurs où tous les éléments stratégiques sont mis en image : les caméras filment les discours, le public, le lieu, les rues adjacentes, mais aussi la place et donc les écrans sur lesquels sont diffusées les images en direct (Sécail, 2016). Ces dernières sont parfois photographiées par le public spectateur. C'est le cas d'un père de famille qui, après avoir essayé plusieurs fois de photographier François Hollande sur la scène, se tourne vers l'écran géant devant nous et fait un zoom sur celui-ci. Il montre la photographie à sa femme et sa fille en déclarant : " on le voit bien, ça rend pas mal !". Les écrans (voir leur placement en figure 5) diffusent fréquemment des images de la place du Capitole. La plupart des séquences qui montrent la foule sont prises de dessus accentuant ainsi l'effet d'unité massive. Dans ces dispositifs, le public en apparence très actif (agitation des drapeaux, cris de ralliements) n'en est pas moins cantonné à un espace d'expression très limité.

Le meeting de Jean-Luc Mélenchon illustre aussi ce phénomène. L'encadrement des comportements des participants procède de deux moments distincts : avant le meeting (appropriation des marqueurs de l'identité politique du candidat pour s'en faire le propre relais dans l'espace public) et pendant le meeting (resserrement des espaces d'expression et (auto-) contrôle des uns sur les autres). Tout d'abord, le choix d'un meeting en plein air permet une relative souplesse de circulation, de tenues des corps (vêtements, gestuels, postures assises au sol, etc.) et d'activités par rapport aux meetings ayant lieu dans des espaces clos. Cette marge de liberté d'appropriation de l'espace du meeting et, plus largement, de l'espace public, est à mettre en relation avec sa temporalité : l'investissement de la place du Capitole par le public débute plusieurs heures avant que le dispositif d'accueil ne soit finalisé (les barrières délimitant l'accès VIP n'ont été installées qu'en fin d'après-midi). La scène est encore vide, les écrans éteints et toute la place en dehors de la scène est accessible. Des bus de militants sont arrivés en début d'après-midi et les passagers se sont déployés dans le centre-ville. Clairement identifiables politiquement par des autocollants sur leurs vêtements ou des pin's de campagne, ils arpentent les rues de manière ostentatoire. Cette forme d'appropriation de l'espace public correspond aux pratiques militantes de ce groupement politique habitué aux manifestations (Ripoll, 2008) comme le montre l'encadré 3.

Encadré 3. La culture politique d'occupation de l'espace public par le Front de gauche

Dès 15h, vers le milieu de la place du Capitole, des militants du Front de gauche organisent un atelier de pancartes et d'affiches. Les pots de peintures et les 
bombes sont à disposition. Des passants réalisent leurs propres pancartes pour le soir et ont pour mission de recruter d'autres passants dans ce même but.

Extrait de carnet de terrain sur la place la place du Capitole le 5 avril 2012 vers $15 \mathrm{~h}$.

Un groupe de sept jeunes qui portent des perruques et des habits de couleurs vives, quelques instants avant l'heure annoncée du meeting de Jean-Luc Mélenchon, chante autour d'une guitare, au rythme de la chanson «Bella ciao ». Ils achèvent par « spéciale dédicace à Toulouse-Nougaro : Toulouse tu n'es pas rose... Tu es toute rouge !». Ils transmettent ainsi autrement le message politique qui sera repris sérieusement sur l'estrade : « le 5 avril, si tu hésites entre Hollande et le candidat Jean-Luc Mélenchon, écoute ton cœur et ta raison et vient voter pour Mélenchon ». Cette appropriation politique, en partie ludique, fait notamment écho au tract distribué par un groupe de jeunes communistes : «Votez utile, poisson d'avril ! ». Ou encore cette fanfare de 8 musiciens qui attire les spectateurs, certains portant ostensiblement l'autocollant, au centre du tee-shirt, «Prenez le pouvoir », pendant que d'autres ont collé sur leur instrument l'étiquette : «Qu'ils s'en aillent tous ». D’autres groupes de jeunes assis sur la place jouent à des jeux de société en buvant de la bière.

Ensuite, lorsqu'arrive l'heure du meeting, les participants se rassemblent sur la place $\mathrm{du}$ Capitole, resserrant ainsi l'espace de la mobilisation. Devant les grilles, mais excentré, un stand d'accueil a été installé pour orienter les gens qui souhaiteraient aider à coller des affiches, distribuer des tracts. Certains fument, discutent, boivent un verre à côté d'autres qui écoutent très sérieusement les premiers intervenants. Ceux-ci sont nombreux et se succèdent ou apparaissent simultanément sur scène. Ils présentent et représentent le mouvement dans sa diversité. Cependant, lorsque vient le tour du candidat, il occupe seul la scène et commence par parler bas afin que le silence se fasse, comme en classe. Avec un ton sérieux et calme, il s'adresse au public seul en scène. Une dame à côté de nous s'exclame "on se croirait à la messe!». La personnification est importante et la posture de l'orateur classique. Elle se confond avec celle observée dans les meetings de François Hollande ou Nicolas Sarkozy. Ainsi, si les corps sont relativement détendus deux ou trois heures avant l'arrivée du candidat, ils sont beaucoup plus (auto-) contrôlés au moment de l'entrée sur scène du candidat : l'espace de mouvement est en effet beaucoup plus réduit lorsque la place enserre des milliers de spectateurs, l'accès est d'ailleurs fermé par des barrières métalliques dans les rues conduisant au Capitole. Là encore, le public est assigné à la seule position de spectateur, délégataire espéré de la voix du candidat. La personnification des candidats et la délégation sont au centre de ce rituel politique tel qu'orchestré par les partis dominants de cette élection. Beaucoup plus bas dans la hiérarchie sondagière, d'autres groupes, à l'extrême gauche de l'échiquier politique proposent une appropriation différente du rite afin qu'il s'accorde à leurs valeurs politiques.

\section{Dépersonnalisation du candidat et valorisation de l'interconnaissance : une gouvernementalité par la proximité}

Les dispositifs mis en place par les partis les plus à gauche de l'échiquier politique donnent une autre vision du meeting, plus proche de la réunion publique. L'occupation 
de l'espace, la présence physique du candidat et sa position avant, pendant et après le meeting, le ton comme la disposition scénique sont organisés de façon à gommer la spécificité de la place du candidat, en accord avec le message politique porté.

Pour autant, l'organisation de l'espace, restreint dans les salles de meeting choisies, «instaure un contrôle [encore plus fort] des militants sur les autres militants" (Baloge, 2016 : 231). Ce contrôle est renforcé, dans le cas du meeting de la candidate de Lutte ouvrière, par la présence d'un service d'ordre particulièrement visible ${ }^{30}$. Nathalie Arthaud poursuit le modèle classique des réunions publiques. L'ambiance de la salle est très studieuse : les participants semblent concentrés sur le discours de la candidate et certains prennent des notes, ce qui apparaît très éloigné de ce que nous avons pu observer dans la grande majorité des autres rassemblements. Il y a très peu de bruit et les réactions du public sont souvent unanimes. Nous sommes frappés par le ton sec et sérieux de la candidate qui, même lorsqu'elle fait des traits d'humour dans son discours, conserve une attitude sévère. Par exemple, lorsqu'elle déclare que «Sarko pourrait se reconvertir comme acteur de série B dans le cinéma ", le public applaudit et agite parfois des drapeaux mais personne ne rit de façon sonore et personne ne parle en même temps que la candidate, sauf quelques militants du NPA regroupés au même rang.

Principalement destiné à des militants locaux, ce meeting se déroule dans une salle gratuite du centre-ville de Toulouse, assez peu visible de la rue, disposant de 400 places assises. L'entre soi est manifestement très fort : à l'arrivée dans la salle, beaucoup de militants sont déjà là et certains sont arrivés avant pour manger ensemble (encadré 4). Dans ce dispositif, la surveillance généralisée, sur le mode de l'interconnaissance, implique une autodiscipline des corps permanente : le regard des autres structure les possibilités de déplacements, les gestes conformes et les expressions du visage. Il n'y a quasiment que des militants et quelques proches notamment des militants du NPA. Les chaises sont bien centrées et alignées en deux parties séparées par une large allée centrale. Ce sentiment d'encadrement, à la fois physique et émotionnel, est renforcé par le cadrage visuel des affiches de campagnes (voir la disposition de la salle en figure 6 , et l'encadré 4) mais aussi par la présence d'un service d'ordre qui domine entièrement l'espace.

Suite à son discours prononcé debout derrière un pupitre où sont posées ses notes, Nathalie Arthaud se rassoit à la table installée sur scène, aux côtés de la responsable locale, pour répondre aux questions de l'assemblée dans un dispositif assez proche d'un enseignement scolaire. C'est le seul dispositif de meeting où la salle peut participer concrètement à la prise de parole politique. Il s'agit là de réduire la distance sans l'annuler, et de présenter la pratique de l'activité politique comme résultant d'une acquisition progressive de connaissances à la portée de chacun. Mais malgré la programmation d'un temps de questions/réponses avec la salle, les interventions de membres du public sont très peu nombreuses et principalement portées par des militants actifs du groupe ${ }^{31}$. Ce contrôle de la parole légitime conduisant à des formes d'auto-exclusion que l'on retrouve notamment chez les militants des petites sections du parti socialiste est à mettre en lien avec l'organisation de l'espace : « du fait de la faible surface du lieu et du matériel militant présent, la pièce est assez chargée, ce qui renforce à la fois le sentiment de proximité entre militants et l'impression que la section est spatialement limitée pour accueillir un nombre restreint de militants » (Baloge, 2016:229). 
Figure 6. Meeting de Nathalie Artaud, salle des allées de Barcelone, 30/03/2012 (20h30)

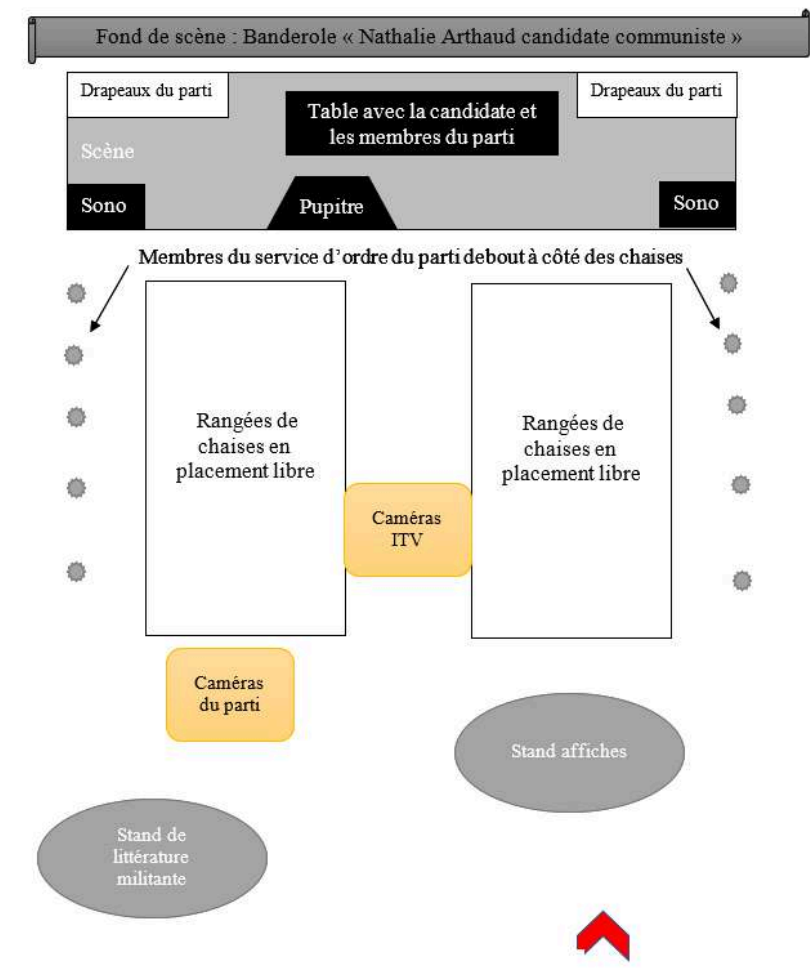

Source : collectif SPEL Meeting toulousain, 12/12/2013.

\section{Encadré 4. Un meeting de convaincus}

Carnet de terrain du 30/03/2012

Il n'est pas attendu plus de 200 personnes au vu des chaises installées et toutes les personnes sur place semblent se connaitre ou se reconnaitre à l'entrée. Celles arrivées comme nous pour l'heure annoncée du meeting s'approchent des tables où le repas s'achève et embrassent des participants. Beaucoup de gens nous disent bonjour et certains nous font la bise, comme s'ils nous connaissaient, comme s'il était évident que parce que nous nous trouvons là, nous faisons partie du groupe.

Le pourtour de la salle est tapissé de sept affiches de campagne collées tous les 1,5 mètres, donnant une impression d'encadrement très fort. À gauche on peut lire « pour s'opposer aux patrons contrôlons leur comptabilité » et à droite simplement « Lutte Ouvrière ». Une table avec de la littérature militante est présente au fond de la salle, côté entrée. L'ensemble de l'espace paraît décliner volontairement le principe de l'austérité et de la rigueur. Une table recouverte d'un tissu rouge et un pupitre accueillent la candidate et sa représentante locale ; derrière se trouve une banderole où est écrit « une candidate communiste ».

Dans un registre proche mais plus festif et détendu, le Nouveau Parti Anticapitaliste valorise le collectif et met en scène la non-professionnalisation et la dépersonnalisation du candidat. Il se donne à voir au public en collectif : le candidat Philippe Poutou se déplace dans la salle avant l'intervention, comme son prédécesseur Olivier Besancenot. 
Ces deux figures du parti offrent une conférence de presse dans le hall d'entrée de la salle peu de temps avant le meeting. Ensuite, pendant que le public s'installe, ils restent ensemble suivis de quelques journalistes qui prennent des photographies. Sur leur passage, ils saluent et discutent avec des militants. La présence de Philippe Poutou ne crée pas d'attroupement et il peut circuler sans difficulté, de façon relativement anodine au regard d'autres candidats évoqués plus haut. La salle a été aménagée de manière à favoriser la convivialité : toutes les chaises n'ont pas été installées (environ 900 sur les 1400 disponibles dans cette salle) et celles qui restent sont utilisées pour délimiter l'espace de réunion de l'espace de divertissement (figure 7).

Figure 7. Meeting de Philippe Poutou, salle Mermoz, 17/04/2012 (20h30)

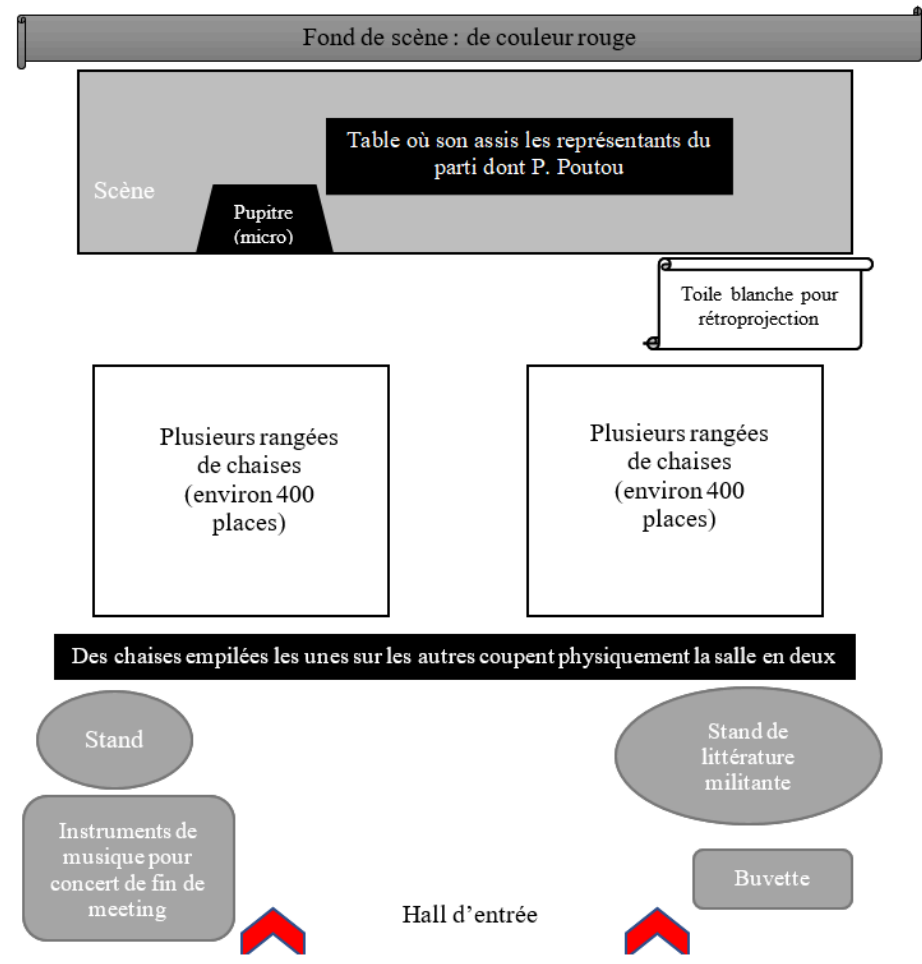

Source : collectif SPEL Meeting toulousain, 12/12/2013.

Pendant le meeting, sur scène, une grande table accueille les cinq intervenants qui sont tous visibles pendant toute la durée du meeting. Chaque intervenant se lève pour aller parler au pupitre. Le candidat n'apparaît donc à aucun moment seul face à la salle. Après une intervention des salariés de Freescale ${ }^{32}$, une présentation des prochains rassemblements par une militante et l'annonce d'une liste aux législatives par un militant (tous assis à la table sur scène), Olivier Besancenot commence un discours au pupitre. Il l'achève en déclarant: " $\mathrm{Ne}$ perdez pas de vue que les gens qui vous représentent le mieux sont parmi vous». Les applaudissements sont réguliers et soutenus mais lorsque Philippe Poutou prend la parole avec un «salut à toutes et à tous ", le discours est moins rythmé et le candidat gère mal les temps de pause et d'applaudissements. Il finira d'ailleurs par tourner cela en auto-dérision en déclarant : « là vous pouvez applaudir! ». Globalement, la salle est bruyante pendant son discours. Le public se déplace, certains n'écoutent que quelques intervenants puis sortent de la salle pour fumer ou échanger en petits groupes à l'extérieur, d'autres restent à la 
buvette. Ici, la focale n'est plus mise sur le candidat, mais sur le dispositif dans son ensemble, qui doit permettre de regrouper, de créer l'échange. La revendication du non professionnalisme est posée comme principe (d'action) politique, faite par tous et pour tous. Là encore, l'organisation formelle donne à voir des principes et valeurs politiques défendus par le groupe. Il s'agit de revendiquer une action politique par et pour des citoyens comme les autres, tout en réussissant à galvaniser les foules afin de soutenir le candidat. Pour autant, la participation effective du public reste très limitée : il n'y a pas de temps d'échange avec la salle et les orateurs sur scène ont été sélectionnés parmi les principaux militants locaux. La remise en cause du dispositif classique du meeting se concentre sur la personnification du candidat plus que sur la délégation qui est au fondement de l'enjeu électoral.

Malgré leur volonté de proposer ou de présenter un dispositif ouvert au public et participatif, les professionnels gardent la main sur le déroulement du meeting et les messages qu'ils veulent faire passer. Le rituel s'inscrit donc, ici aussi, dans une gouvernementalité spatiale reproduisant les mécanismes de différenciation des professionnels et des profanes du champ politique.

\section{Conclusion}

Analyser l'organisation spatiale des meetings électoraux présente au moins deux intérêts. Tout d'abord, d'un point de vue méthodologique, l'attention prêtée aux espaces et à leurs agencements, dans le temps long du meeting (avant, pendant et après), permet de rendre compte de la diversité de ce rituel politique. Qu'il soit facile ou difficile d'accès, d'un abord festif, accueillant ou plutôt intimidant presque répulsif, le meeting électoral présente cette particularité d'être un événement pensé, organisé et agencé de manière à diffuser un message politique au-delà des militants engagés, tout en gardant la main sur son déroulement. Pour cela, il s'agit d'éviter les désordres et de sécuriser l'événement par le déploiement d'un service d'ordre plus ou moins visible et/ ou disciplinaire. Le dispositif mis en place par les organisateurs du meeting peut alors être appréhendé en tant que disciplinarisation conçue pour que chacun reste à sa place sans déborder et sans contrevenir ni à l'ordre public, ni à l'ordre social ou politique incarné par le parti organisateur. Les modalités du maintien de l'ordre diffèrent selon les tendances politiques mais aussi selon la place du parti dans la compétition électorale. Cela permet de rappeler que la politique se joue aussi matériellement dans le meeting et dans la spatialité de sa mise en ordre, d'où l'intérêt d'intégrer cette dimension aux méthodes d'enquêtes portant sur les meetings électoraux.

Deuxièmement, d'un point de vue analytique, l'étude des meetings dans leur dimension spatiale rappelle que les transformations du rapport au politique et à la politique ne se traduisent pas en un assouplissement de leur encadrement. Contrairement à l'évolution contemporaine en France du contrôle des manifestations de rue (Filleule, Jobard, 2020), si le maintien de l'ordre par la discipline semble euphémisé dans le cadre des meetings électoraux, la mise en scène et en ordre du corps social tenu à distance des professionnels de la politique est bien présente quelle que soit la couleur politique. Cela témoigne d'une forme de pouvoir des institutions électorale et partisane au sens où Michel Foucault l'entendait, soit un pouvoir qui «ne s'exerce pas in abstracto, mais toujours dans un lieu déterminé sur des corps ciblés et selon des modalités définies » 
(Memmi, Guillo, Martin, 2009) et qui se caractérise ici par un gouvernement spatial des conduites.

\section{BIBLIOGRAPHIE}

ABELES M. (2010), « Rituels et communication politique moderne », in Yannic A., Le rituel, Paris, CNRS éd., pp. 61-81.

AUYERO J. (2005), «L'espace des luttes. Topographie des mobilisations collectives », Actes de la recherche en sciences sociales, vol. no 160, no 5, pp. 122-132.

BAAMARA L., FLODERER C., POIRIER M. (dir.) (2016), Faire campagne, Paris, Karthala.

BALOGE M, (2016) «Contrôler le débat militant en section. L'organisation spatiale des réunions au Parti socialiste. » in Combes H., Garibay D., Goirand C., dir. (2016), Les lieux de la colère. Occuper l'espace pour contester, de Madrid à Sanaa, Paris, Karthala, pp. 220-240.

BEAUGUITTE L. (2019), « (Re)politiser l'espace public. Les Nuits debout de quartier du Nord-est parisien (2016-2017) », Carnets de géographes, no. 12, [En ligne], URL : http://

journals.openedition.org/cdg/4745.

BOURDIEU P. (1979), La Distinction. Critique sociale du jugement, Paris, Les éditions de Minuit.

BOURDIEU P. (1982), « Les rites comme actes d'institution », Actes de la recherche en sciences sociales, vol. 43 , no. 1 , pp. 58-63.

BOURDIEU P. (1981), « La représentation politique. Eléments pour une théorie du champ politique ", Actes de la Recherche en Sciences Sociales, vol. 36-37 pp. 3-24.

BOURDIEU P., THOMPSON J. B. (2001), Langage et pouvoir symbolique, Paris, Éditions du Seuil.

BURGER M., JACQUIN J., MICHELI R. (2011), La parole politique en confrontation dans les médias, Louvain-la-Neuve, De Boeck Supérieur.

COSSART P. (2010), Le meeting politique, de la délibération à la manifestation (1868-1939), Rennes, Presses Universitaires de Rennes.

COMBES H. (2009), « Meetings de fin de campagne au Mexique et ethnographie des milieux partisans », Politix, vol. 85, no. 1, pp. 149-179.

COMBES H., GARIBAY D., GOIRAND C. (dir.) (2016), Les lieux de la colère. Occuper l'espace pour contester, de Madrid à Sanaa, Paris, Karthala.

COMBY J-B, GROSSETETE M. (2012), « "Se montrer prévoyant” : une norme sociale diversement appropriée », Sociologie, vol. 3, no. 3, pp. 251-266.

COURTY G., GERVAIS J. (dir.) (2016), Le Lobbying électoral. Groupes en campagne présidentielle (2012), Villeneuve d'Ascq, Presses universitaires du Septentrion.

DESRUMAUX C., LEFEBVRE R. (2016), « Pour une sociologie des répertoires d'actions électorales », Politix, vol. 113, no. 1, pp. 5-16.

FILLIEULE O., JOBARD F. (2020), Politiques du désordre, Paris, Seuil. 
FOUCAULT M. (1975), Surveiller et punir. Naissance de la prison, Paris, Gallimard.

FOUCAULT M. (1994), Dits et Écrits, Tome 3, Paris, Gallimard.

FOUCAULT M. (2004), Sécurité, Territoire, Population. Cours au Collège de France. 1977-1978, Paris, Seuil.

FRANÇOIS A., PHELIPPEAU E. (2015), « Chapitre 3. Le financement des élections en France », Le financement de la vie politique, Paris, Armand Colin, pp. 95-140.

FRETEL J. (2004), « Quand les catholiques vont au parti. De la constitution d'une illusio paradoxale et du passage à l'acte chez les « militants » de l'UDF », Actes de la recherche en sciences sociales, vol. 155 , no. 5, pp. 76-89.

GAXIE D. (1977), « Economie des partis et rétribution du militantisme », Revue française de science politique, vol. 27, no. 1, pp. 123-154.

GAXIE D. (2004), La démocratie représentative, 4e édition, Paris, Montchrestien.

HAFFAF O., NOLLET J. (2013), « La coproduction de l'événement politique. Le travail journalistique et la médiatisation des meetings électoraux de 2012 ", Congrès AFSP, Paris. Section Thématique 24 : Un retour des meetings électoraux ? Les meetings dans la campagne présidentielle: dispositifs, acteurs et publics, Document de travail en ligne : http://www.afsp.info/congres2013/st/ st24/st24haffafnollet.pdf

HMED C. (2008), « Des mouvements sociaux "sur une tête d'épingle” ? Le rôle de l'espace physique dans le processus contestataire à partir de l'exemple des mobilisations dans les foyers de travailleurs migrants ", Politix 84, no 4, 145-65.

HMED C. (2020), « Espace géographique et mouvements sociaux », In Fillieule O., Mathieu L. et Péchu C. (dir.), Dictionnaire des mouvements sociaux, Paris: Presses de Sciences Po, pp. 237-244

LACROIX B., LAGROYE J.(dir.) (1992), Le Président de la République : usages et genèses d'une institution, Paris, Presses de la Fondation nationale des sciences politiques.

LE BART C. (1998), Le discours politique, Paris, Presses Universitaires de France.

MARIOT N. (1995a), « Le rite sans ses mythes : forme rituelle, temps et histoire », Genèses, vol. 21, no. 1 , pp. 148162.

MARIOT N. (1995b), « "Propagande par la vue”. Souveraineté régalienne et gestion du nombre dans les voyages en province de Carnot (1888-1894)», Genèses, vol. 20, pp. 24-47.

MARIOT N. (2001), « Les formes élémentaires de l'effervescence collective, ou l'état d'esprit prêté aux foules ", Revue française de science politique, vol. 51, no. 5, pp. 707-738.

MEMMI D., GUILLO D., MARTIN O. (2009), La tentation du corps : corporéité et sciences sociales, Paris, École des hautes études en sciences sociales.

MERRY S. E. (2001) « Spatial Governmentality and the New Urban Social Order: Controlling Gender Violence Through Law », American Anthropologist, vol. 103, no. 1, pp.16-29.

NEVEU E. (2011), Sociologie des mouvements sociaux, Paris, la Découverte.

POURCHER Y. (1990), « “Un homme une rose à la main” ”, Terrain, no. 15, pp. 77-90.

POURCHER Y. (2004), Votez tous pour moi ! Les campagnes électorales de Jacques Blanc en LanguedocRoussillon (1986-2004), Paris, Presses de Sciences Po.

REGNIER F., MASULLO A. (2009), « Obésité, goûts et consommation. Intégration des normes d'alimentation et appartenance sociale ", Revue française de sociologie, vol. 50, no. 4, pp. 747-773. 
RIPOLL F. (2005), « S'approprier l'espace... ou contester son appropriation ? », Norois, vol. 195, no. 2, [En ligne], URL : http://journals.openedition.org/norois/489.

RIPOLL F. (2008), « Espaces et stratégies de résistance : répertoires d'action collective dans la France contemporaine ", Espaces et sociétés, vol. 134, no. 3, pp. 83-97.

SOMMIER I. (1993), « La CGT : du service d'ordre au service d'accueil », Genèses, vol. 12, pp. 69-88.

SECAIL C. (2016), « Les meetings de la présidentielle 2012. Un dispositif technique et stratégique pour faire campagne », Politiques de communication, vol. 2, pp. 79-109.

SECAIL C. (2020), Les meetings électoraux. Scènes et coulisses de la campagne présidentielle de 2017, Villeneuve d'Asc, Presses universitaires du Septentrion.

SPEL Collectif sociologie politique des élections (2014), Les sens du vote. Une enquête sociologique (France 2011-2014), Rennes, Presses universitaires de Rennes.

TILLY C. (1984), « Les origines du répertoire d'action collective contemporaine en France et en Grande-Bretagne », Vingtième Siècle. Revue d'histoire, vol. 4, no 1, pp. 89-108.

\section{NOTES}

1. Ces tensions se solderont d'ailleurs la nuit suivante par un blessé grave : voir par exemple « Le mauvais sort de l'étudiant chilien » in LibéToulouse.fr, le 9/04/2012.

2. Ces éléments sont issus des carnets d'observation des deux autrices dans le cadre de la recherche collective du groupe «SPEL meeting » toulousain (voir encadré 1).

3. Nous tenons à adresser tous nos remerciements aux relecteur.ice's qui nous ont patiemment accompagnées pour aboutir à ce texte dont pour autant nous assumons seules le contenu et les imperfections. Merci aussi au groupe SPEL meeting, les collègues et ami-e·s du groupe toulousain (Ludivine Balland, Elise Cruzel, Sandra Vera Zambrano, Fanny Touraille, Gildas Hivert, Frédéric Nicolas, Jérémie Nollet, Omar Haffaf) et d'ailleurs (Julien Fretel et Nathalie Ethuin) qui ont permis que cette stimulante enquête collective advienne.

4. Cette recherche a donné lieu notamment à une communication portant sur la médiatisation des meetings électoraux (Haffaf, Nollet, 2013) et à une section thématique consacrée aux meetings électoraux dirigée par Paula Cossart, Ludivine Balland et Elise Cruzel dans le cadre du Congrès de l'Association française de science politique (AFSP) de 2013.

5. Pour Daniel Gaxie, la représentation politique est un espace de compétition et les "professionnels de la représentation politique» sont caractérisés par leur investissement spécifique c'est-à-dire s'appuyant sur une compétence spécifique, une activité publique, un point de vue réflexif sur le monde. Ils donnent un sens politique aux événements et situations.

6. Nous nous appuierons sur cette notion d'« espace public idéal » tout au long du texte. Pour alléger la rédaction, la référence au travail de Paula Cossart ne sera plus systématiquement mentionnée.

7. Voir en particulier dans la préface de Daniel Gaxie (Courty, Gervais, 2016) les pages 13-14 qui reviennent sur ce grand projet, ses réussites et ses déconvenues.

8. Cf. les multiples présentations qui ont eu lieu lors des Journées d'études les 20/21 Mars 2013 : «Les meetings dans les campagnes électorales : analyses sociales historiques et juridiques d'un dispositif politique ", à Sciences-Po Toulouse.

9. Une première communication sur ce thème ayant été réalisée dans le cadre du $12^{\mathrm{e}}$ congrès national de l'Association française de science politique (section thématique 24, 9-11 juillet 2013) à trois avec Gildas Hivert. 
10. Ces estimations permettent de se rendre compte de l'affluence afin de prendre la mesure des choix de dispositifs spatiaux en fonction du nombre de personnes à gérer.

11. Propos recueillis par l'une des enquêtrices pendant l'observation de l'installation de la salle la veille du meeting, auprès de membres de l'organisation nationale du FN. Comme le rappelle Claire Sécail, ce coût d'entrée est un trait distinctif historique de ce parti (Sécail, 2016 : 91).

12. Tous les propos sont recueillis avant, pendant ou après le meeting lors d'échanges où les enquêteurs sont "à couvert », c'est-à-dire qu'ils se présentent comme des participants comme les autres, sans dévoiler les raisons de leur présence. Au cours de discussions informelles avec de jeunes membres du FN, il est mentionné par certains d'entre eux que des membres du services d'ordre extérieurs cherchent l'affrontement avec les opposants.

13. Selon les propos recueillis auprès de membres de l'organisation la veille de l'événement, au FN, le service d'ordre salarie des militants qui sont aussi, pour certains d'entre eux, des professionnels de la sécurité tels que des militaires, mais plutôt peu/pas gradés, des salariés de la sécurité privée ou des convoyeurs de fond.

14. L'équipe d'enquête étant essentiellement féminine ce jour-là, l'invective sous forme de boutade reste un rappel à l'ordre politique et à l'ordre de genre.

15. Selon des propos recueillis auprès de responsables du service d'ordre le jour du meeting.

16. Ce qui est un enjeu important dans le choix des villes comme le rappelle Claire Sécail pour la campagne présidentielle de 2017 (Sécail, 2020 : 98-99).

17. C'est le cas d'une des enquêtrices, coincée dans les bouchons dès la sortie de la rocade qui, sur les conseils des collègues déjà sur place et bloqués à l'extérieur de la salle (5 enquêteurs sur place) décide de rebrousser chemin. Parmi les autres enquêteurs aucun ne parviendra à pénétrer jusque dans la salle, un étant resté dehors et quatre dans le hall d'entrée.

18. Nous sommes entrés sans être fouillés et c'est dans une bousculade dangereuse que nous nous sommes entassés devant la porte.

19. Pas de lieu de vente de boissons et l'accès aux toilettes était quasi impossible en raison du monde agglutiné dans l'entrée.

20. Propos notés par l'enquêteur resté sur le parking devant la salle, qui a pu interroger à la volée quelques militants et sympathisants sortants du meeting puis, une fois la salle évacuée, quelques responsables de l'organisation restés à l'intérieur pour ranger.

21. Les informations concernant la présence d'un de ces agents auprès de Jean-Luc Mélenchon sont assez contradictoires, laissant planer le doute sur le fait qu'il ait accepté la présence d'un de ces agents au cours de la campagne ou qu'il l'ait refusée jusqu'au bout. Philippe Poutou a, pour sa part, refusé. Nathalie Artaud, tout comme Jacques Cheminade et Nicolas Dupont-Aignan ne semblent pas en bénéficier.

22. Extrait du discours du responsable du service d'ordre du Front de Gauche, propos recueillis durant la réunion de préparation avec des volontaires, le 5 avril 2012 à $15 \mathrm{~h}$.

23. Les autrices ont participé avec d'autres membres de l'équipe à cette réunion. Présentes sur la place du Capitole dès le début d'après-midi pour observer les stands de création de pancartes ouverts au public par des militants, elles ont suivi des groupes qui se dirigeaient vers une salle à proximité de la place. Elles sont entrées sans que personne ne leur demande rien et ont pu assister à la réunion dans son intégralité avant de rejoindre le meeting le soir même.

24. Le Front de Gauche est le produit d'une coalition de groupes dont des partis : Parti de Gauche, Parti Communiste, plusieurs fractions du NPA qui sont arrivées successivement, des collectifs issus de la tentative de campagne unitaire à la présidentielle de 2007 à la suite du Non au référendum sur le traité européen de 2005 et de la candidature de José Bové à l'élection présidentielle de 2007.

25. Rhétorique de la FI durant cette campagne. 
26. Information fournie par Julien Fretel lors de la Journée d'étude sur les meetings à Toulouse les 20 et 21 mars 2013.

27. Lorsqu'elle accuse la classe politique de ne pas respecter la constitution, elle lit un extrait de cette constitution sur un papier puis le déchire en disant, « ils sont en train de la déchirer sous les yeux du peuple français ".

28. Cette manière de dispenser son discours, héritée de son père, reste une exception dans les meetings de campagne, la plupart des candidats étant plutôt debout, statique, derrière un pupitre (Pourcher, 1990)

29. Pour une description et une tentative d'analyse des choix musicaux dans les meetings, se référer au travail de Claire Sécail sur les "ambiances musicales" dans les meetings de la campagne présidentielle de 2017 (2020:114).

30. Et qui n'est pas sans rappeler la vision de la gestion de l'ordre que nous avons décrite en première partie concernant les partis de droite, nous y reviendrons.

31. Ceux qui nous avaient accueillis et étaient présents dans la salle en amont.

32. Une entreprise américaine ayant décidé la fermeture de son usine toulousaine en 2012.

\section{RÉSUMÉS}

Dans cet article consacré aux meetings politiques organisés dans le cadre de la campagne pour l'élection présidentielle de 2012, nous proposons de tourner le regard vers la salle plutôt que vers la scène, et vers le dispositif en lui-même plutôt que vers le candidat. Cet article s'empare ainsi de la dimension spatiale comme paramètre central des modes de contrôle du public par les organisateurs du meeting. L'organisation spatiale des meetings matérialise également une vision politique de la société propre à chaque parti politique. L'article montre en effet comment les répartitions du public et les différentes modalités de contrôle des conduites des participants incarnent des conceptions variées de l'espace public idéal. Cette étude s'appuie sur l'observation de huit meetings qui se sont déroulés à Toulouse pendant la campagne présidentielle de 2012 dans le cadre de l'enquête nationale SPEL.

This article deals with the political meetings organized during the 2012 French presidential election. We intend here to look away from the stage and rather to focus on the rooms and devices. This article tackles the spatial dimension as a central explanatory element in order to understand the modalities of public control deployed by the organizers of the meetings. The spatial organization of the meetings also materializes a political vision of society specific to each political party. The article shows how the distribution of the public and the different ways of controlling the conduct of the participants embody various conceptions of the "ideal public space". This study is part of the national SPEL survey and is based on the observation of eight meetings that took place in Toulouse during the 2012 presidential campaign.

\section{INDEX}

Thèmes : Carnets de recherches

Keywords : meeting, order, spatial governmentality, discipline, control

Mots-clés : meeting, ordre, gouvernementalité spatiale, discipline, contrôle 


\section{AUTEURS}

\section{AÏCHA BOURAD}

Odycée, UMR AGIR, INRA Toulouse

aicha.bourad[at]inra.fr

\section{FANNY PARENT}

LaSSP, IEP Toulouse.

Parent.fanny[at]gmail.com 